High Energy Phenomena in Relativistic Outflows III (HEPRO III)

International Journal of Modern Physics: Conference Series

Vol. 8 (2012) 404-407

(C) World Scientific Publishing Company

DOI: 10.1142/S2010194512005004

\title{
THE HIGH-ENERGY PROPERTIES OF 3C 454.3: A FOUR-YEAR STUDY
}

\author{
STEFANO VERCELLONE \\ INAF Istituto di Astrofisica Spaziale e Fisica Cosmica \\ Via Ugo La Malfa, 153 - I-90146 Palermo, Italy \\ stefano.vercellone@iasf-palermo.inaf.it \\ on behalf of the AGILE Team
}

\begin{abstract}
The blazar 3C 454.3 has become the most active and brightest $\gamma$-ray source of the sky, earning the nickname of Crazy Diamond. The short-term variability in the $\gamma$-ray energy band and the extremely high peak fluxes reached during intense flaring episodes make 3C 454.3 one of the best targets to investigate the blazar jet properties. We will review four years of observational properties of this remarkable source, discussing both short- and long-term multi-wavelength campaigns, with particular emphasis on the recent flaring episode which occurred on 2010 November 20, when 3C 454.3 reached on a daily time-scale a gamma-ray flux $(E>100 \mathrm{MeV})$ higher than $6 \times 10^{-5}$ photons $\mathrm{cm}^{-2} \mathrm{~s}^{-1}$, about six times the flux of the brightest $\gamma$-ray steady source, the Vela Pulsar.
\end{abstract}

Keywords: galaxies: active - galaxies: quasars: general - galaxies: quasars: individual: 3C 454.3 - galaxies: jets - radiation mechanism: non thermal.

\section{The $\gamma$-ray blazar $3 \mathrm{C} 454.3$}

The blazar 3C 454.3 (PKS 2251+158; $z=0.859$ ) is a well-known $\gamma$-ray flat spectrum radio quasar which exhibits high dynamic range flux variations from the radio to the $\gamma$-ray energy bands. A study ${ }^{1}$ showed that during the EGRET era this source exhibited the highest $\gamma$-ray activity index value, despite moderate $\gamma$-ray peak fluxes $\left(F_{\mathrm{E}>100 \mathrm{MeV}}<2 \times 10^{-6}\right.$ photons $\left.\mathrm{cm}^{-2} \mathrm{~s}^{-1}\right)$. In 2005 May 3C 454.3 exhibited an extremely intense flare at different energies ${ }^{2-5}$, but no information on the $\gamma$-ray flux level was available. The launches of the AGILE ${ }^{6}$ and Fermi $^{7} \gamma$-ray satellites allowed a tremendous improvement in the monitoring of blazars in the $\gamma$-ray energy band, thanks to their wide field of view and all-sky scanning pointing mode ${ }^{\mathrm{a}}$. Moreover, we obtained almost simultaneous SEDs of 3C 454.3 both in the low and and in the high emission levels through dedicated blazar observations performed by the WEBTGASP Consortium ${ }^{8}$ (radio, optical, and NIR), and, thanks to its extremely rapid repointing capability, by Swift $^{9}$ (optical, UV, X-rays, and hard X-rays). Here we

aThe AGILE satellite switched from a pointing to a spinning observing mode on 2009 November because of a permanent reaction wheel failure. 
present the results of four years of AGILE $\gamma$-ray and multi-wavelength observations of 3C 454.3, discussing both its short- and long-term properties.

\section{Long-term monitoring}

During the period 2007 July - 2009 January AGILE detected 3C 454.3 in high $\gamma$-ray state several times. The follow-up multi-wavelength campaigns ${ }^{10-13}$ allowed us to obtain important information on the location of the $\gamma$-ray emitting zone, on the possible time-lags between the flux variations at different energies, and on the physical properties of the jet by means of a detailed SED modeling. Figure 1, left panel (adapted from Ref. 13), shows the $R$-band (top), the mm-230 GHz (middle), and the $\gamma$-ray $E>100 \mathrm{MeV}$ (bottom) light curves. Detailed investigation by means of the cross-correlation function of the correlations between the optical and the $\gamma$ ray light curves in different flaring episodes yielded a correlation between the two energy bands, with a possible lag of the $\gamma$-ray flux variations with respect to the optical ones of about half a day. The different variability behavior of the three light curves can be understood in terms of a change in orientation of a curved jet, yielding different alignment configurations within the jet itself (see Refs. 15-16 for details on the model). Another result of this long-term monitoring is shown in Figure 1, right panel. Black symbols show a clear harder-when-brighter trend in the Swift/XRT data. Nevertheless, this results is quite different when compared with the trends observed during the 2009 December ${ }^{17}$ (green symbols) and the 2010 November $^{18}$ (red symbols) $\gamma$-ray super-flares, as discussed below. The (leptonic) modeling of several SEDs accumulated during this 18-months period at different $\gamma$-ray flux levels
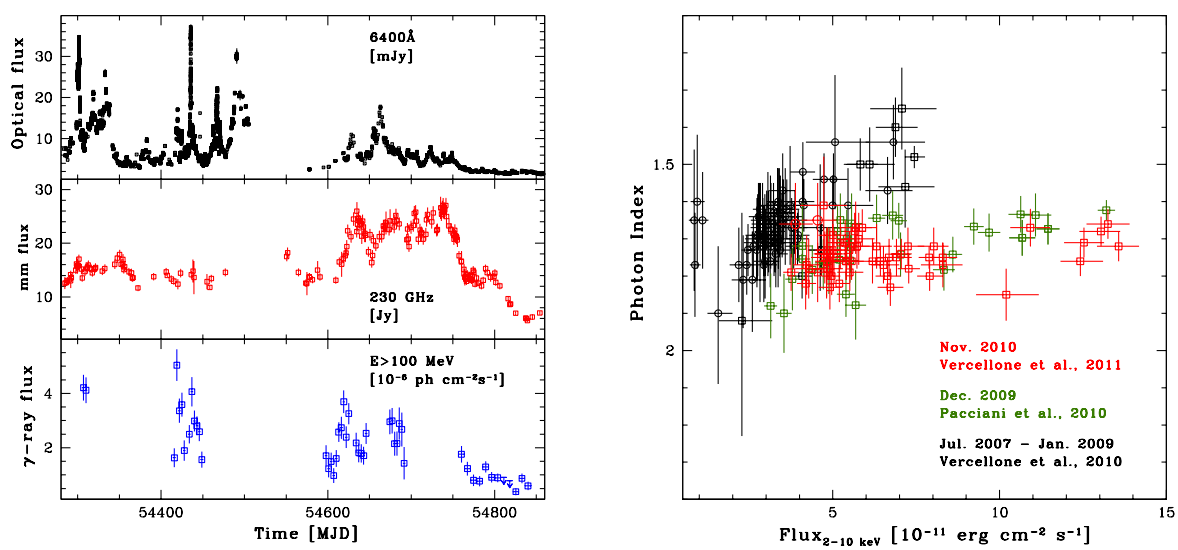

Fig. 1. Left panel: $R$-band, $230 \mathrm{GHz}$, and $\gamma$-ray light curves (top, middle and bottom panel, respectively) covering the period 2007 July - 2009 January. The AGILE light-curve has a time-bin of 1-week. Right panel: Swift/XRT photon index as a function of the $2-10 \mathrm{keV}$ flux. Open circles and squares refer to photon counting and windowed data, respectively (see Ref. 14). 
shows that during the bright flares the $\gamma$-ray emission can be interpreted as inverse Compton scattering of external photons from the broad line region (BLR) clouds off the relativistic electrons in the jet. Moreover, the thermal signature of the accretion disk can be detected during low $\gamma$-ray states.

\section{Short-term monitoring}

Among the several $\gamma$-ray flares detected so far, two stand out for their particularly intense emission and duration. In both cases the $\gamma$-ray flux level well exceeded the one of the most intense $\gamma$-ray steady source, the Vela pulsar. The first one occurred on 2009 December 2-3 when 3C 454.3, after an enhanced $\gamma$-ray state lasted about 2 weeks, displayed an extremely fast flux increase up to $F_{\mathrm{E}>100 \mathrm{MeV}}=$ $(2.0 \pm 0.4) \times 10^{-5}$ photons $\mathrm{cm}^{-2} \mathrm{~s}^{-1}$, as reported in Ref. 19. A multi-wavelength campaign $^{17}$ showed that while the pre- and post-flare SEDs can be modeled in terms of one-zone synchrotron self-Compton (SSC) plus external Compton emission (EC), the flare one requires an additional particle component.

The second $\gamma$-ray super-flare from 3C 454.3 occurred on 2010 November 20 , when $3 \mathrm{C} 454.3$ reached a $\gamma$-ray peak flux of $F_{\mathrm{E}>100 \mathrm{MeV}}=(6.8 \pm 1.0) \times$ $10^{-5}$ photons $\mathrm{cm}^{-2} \mathrm{~s}^{-1}$, as reported in Ref. 18. Figure 2 (left panel) shows the multi-wavelength light curves of this extremely intense flare. While on MJD 55520 a clear peak is visible in all the energy bands, about ten days earlier a remarkably fast optical flare (with rise and fall of about a factor of 2-2.5 in about 48 hr) occurred, with no significant variation in the $\gamma$-ray energy band and a modest $20 \%$ increase in the X-ray flux. Figure 2 (right panel, adapted from Ref. 18)
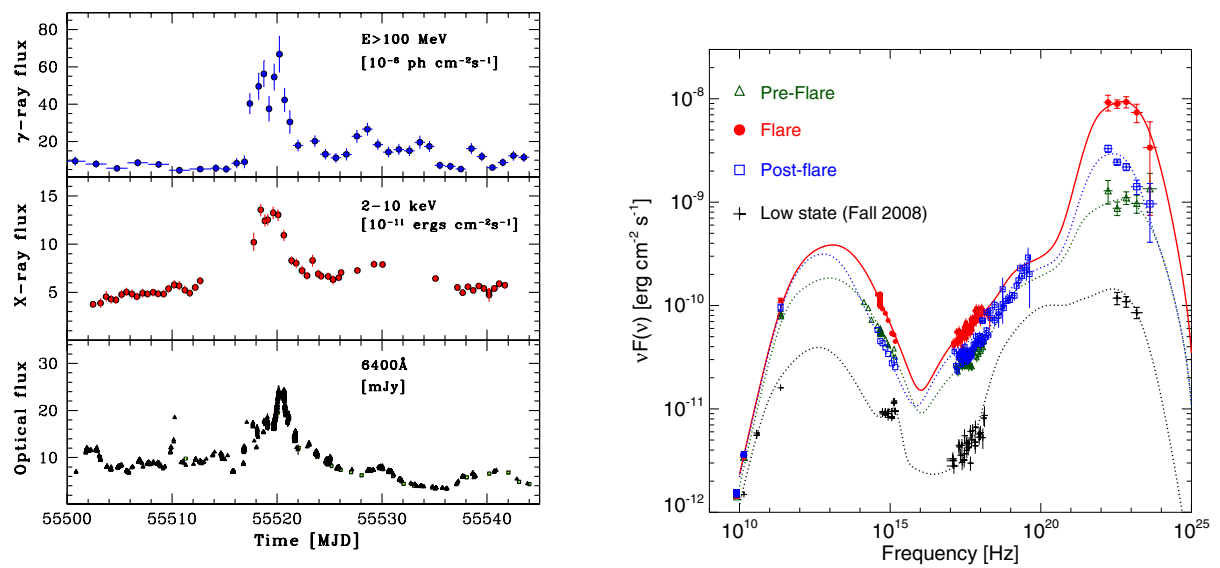

Fig. 2. Left panel: 2010 November $\gamma$-ray, X-ray, and $R$-band light curves (top, middle and bottom panel, respectively). The AGILE light-curve has a variable time bin of $\approx 2,1$, and 0.5 days. Right panel: SEDs accumulated during the 2010 November flare (in colors) compared with a SED accumulated during a particularly low $\gamma$-ray state in Fall 2008 (in black). 
shows the SEDs accumulated before, during, and after the 2010 November $\gamma$-ray flare (color points) in comparison with the SED accumulated in the Fall 2008 during a particularly low state. A modeling of the evolution of the super-flare SED, taking into account the so-called "orphan" optical flare on MJD 55510, challenges a model with a uniform external photon field. Moreover, the modeling places the $\gamma$-ray emission region within the BLR. By combining the Swift/XRT data accumulated during both the 2009 December and 2010 November campaigns with those of the long-term campaign (see Figure 1), we argue that during the $\gamma$-ray super-flares there is a substantial balance between the SSC and the EC(disk) emissions (which results in a quasi-achromatic increase of the $2-10 \mathrm{keV}$ flux), while during much less energetic $\gamma$-ray flares the $\mathrm{EC}($ disk) emission is dominant over the $\mathrm{SSC}$ one.

\section{Conclusions}

The AGILE $\gamma$-ray observations of $3 \mathrm{C} 454.3$, in conjunction with almost simultaneous multi-wavelength campaigns, allowed us to study this source in great details. Nevertheless, the intimate mechanisms responsible for the remarkable $\gamma$-ray fluxes during these super-flares are not yet completely understood.

\section{Acknowledgments}

We acknowledge financial contribution from the agreement ASI-INAF I/009/10/0.

\section{References}

1. S. Vercellone et al., Mon. Not. R. Astron. Soc. 353, 890 (2004).

2. L. Fuhrmann et al., Astron. Astrophys. 445, L1 (2006).

3. E. Pian et al., Astron. Astrophys. 449, L21 (2006).

4. M. Villata et al., Astron. Astrophys. 453, 817 (2006).

5. P. Giommi et al., Astron. Astrophys. 456, 911 (2006).

6. M. Tavani et al., Astron. Astrophys. 502, 995 (2009).

7. W. B. Atwood et al., Astrophys. J. 697, 1071 (2009).

8. M. Villata et al., Astron. Astrophys. 424, 497 (2004). (www.oato.inaf.it/blazars/webt)

9. N. Gehrels et al., Astrophys. J. 611, 1005 (2004).

10. S. Vercellone et al., Astrophys. J. 676, L13 (2008).

11. S. Vercellone et al., Astrophys. J. 690, 1018 (2009).

12. I. Donnarumma et al., Astrophys. J. 707, 1115 (2009).

13. S. Vercellone et al., Astrophys. J. 712, 405 (2010).

14. D. N. Burrows et al., Space Sci. Rev. 120, 165 (2005).

15. M. Villata et al., Astron. Astrophys. 504, L9 (2009).

16. C. M. Raiteri et al., Astron. Astrophys. in press (2011), [arxiv:1107.1093].

17. L. Pacciani et al., Astrophys. J. Lett. 716, L170 (2010).

18. S. Vercellone et al., Astrophys. J. Lett. 736, L38 (2010).

19. E. Striani et al., Astrophys. J. 718, 455 (2010). 